Check for updates

Cite this: RSC Adv., 2018, 8, 33681

\title{
Degradation of dichloroacetonitrile by a UV/ peroxymonosulfate process: modeling and optimization based on response surface methodology (RSM)
}

\author{
Xing Zhang, ${ }^{a}$ Jilun Yao, (D) ${ }^{* b}$ Wei Peng, ${ }^{a}$ Wensi Xu, ${ }^{a}$ Zhongguo Li, ${ }^{a}$ Chong Zhou ${ }^{a}$ \\ and Zhendong Fang*a
}

Response surface methodology (RSM) was utilized to model and optimize the dichloroacetonitrile (DCAN) degradation process by UV/PMS. A quadratic function between DCAN degradation efficiency and three factors including dosage of PMS, UV power and retention time was obtained. The model fitted very well according to high the value of $R^{2}(0.9919)$ and $\mathrm{Adj}-R^{2}$ (0.9814). Additionally, the analysis of variance showed the influence of factors on degradation efficiency followed: retention time $>$ UV power > dosage of PMS. Finally, the optimum conditions were suggested under this model. The degradation efficiency reached the maximum value of $96.2 \%$ with the optimum conditions: dosage of PMS $=0.2 \mathrm{mM}, \mathrm{UV}$ power $=7.95 \mathrm{~W}$, retention time $=80 \mathrm{~min}$.

Received 21st August 2018

Accepted 25th September 2018

DOI: $10.1039 / \mathrm{c} 8 \mathrm{ra07009a}$

rsc.li/rsc-advances

\section{Introduction}

With the first discovery of trihalomethanes in drinking water in $1974,{ }^{1}$ the risk of disinfection by-products (DBPs) in drinking water has attracted much attention. Compared to conventional carbonaceous disinfection by-products (C-DBPs) such as trihalomethanes and haloacetic acids, nitrogen-containing disinfection by-products (N-DBPs), especially HANs, have received more attention in recent years due to their greater toxicity to mammals. The genotoxicity of HAN is the strongest of all DBPs, and its cytotoxicity is 200 times higher than that of haloacetic acid. ${ }^{2}$

Some scholars have measured the N-DBP concentration of drinking water of twelve water treatment plants in the United States, and found that the concentration of HANs was the highest, reaching $14 \mu \mathrm{g} \mathrm{L}^{-1}$. $^{3}$ In Izmir, Turkey, the total HAN concentration of tap water was measured and reached 88.4 $\mu \mathrm{g} \mathrm{L}{ }^{-1}{ }^{4}$ Due to the extremely high toxicity of HANs, the World Health Organization makes a guideline for the limits of HANs in drinking water: the concentration of DBAN and DCAN should not be more than $70 \mu \mathrm{g} \mathrm{L}^{-1}$ and $20 \mu \mathrm{g} \mathrm{L}^{-1}$, respectively. ${ }^{5}$ In the measurement results of HANs in some drinking waters, the concentration level of DCAN is the highest, exceeding $90 \%$ of the total HAN concentration. ${ }^{3,4}$ As a result, it is meaningful and important to control HANs (especially DCAN) in drinking water.

${ }^{a}$ Department of Military Facilities, Army Logistics University of PLA, Chongqing 401331, China

${ }^{b}$ Engineering and Technological Research Center of National Disaster Relief Equipment, Army Logistics University of PLA, Chongqing, 401331, China. E-mail: zing@whut.edu.cn
According to previous studies, the control of HANs could be mainly divided into three strategies: (1) removing the HANs precursors in the water before disinfection; (2) studying the formation mechanism of HANs in the chlor(am)ination disinfection process, and optimizing the formation process to reduce the amount of HANs generated; ${ }^{6}$ (3) directly removing HANs after chlor(am)ination. ${ }^{7}$ Although the first two methods have certain effects on the control of HANs in drinking water, HANs inevitably formed in the water distribution system due to the presence of residual chlorine. Therefore, the direct removal of HANs (not for their precursor) has the potential advantage for HANs control. Some studies have proved the high efficiency for removing HANs ${ }^{7,8}$ However, these studies often focused on the mechanism and theoretical research of reaction process. There has been no report on the modeling or optimization the degradation process for application in reality.

In this paper, RSM was utilized to model and optimize the dichloroacetonitrile (DCAN) degradation process by UV/PMS. Moreover, the effects and interactions of factors including dosage of PMS, UV power and retention time were investigated using the experiment design methodology. Finally, the optimized condition of the process was proposed and verified.

\section{Material and methods}

\subsection{Chemical}

Peroxymonosulfate $\left(\mathrm{KHSO}_{5} \cdot 0.5 \mathrm{KHSO}_{4} \cdot 0.5 \mathrm{~K}_{2} \mathrm{SO}_{4}\right)$ and methyl $t$ butyl ether (MTBE) were purchased from Aladdin. DCAN was obtained from Macklin Biochemical Co., Ltd. Sodium dihydrogen phosphate and dibasic sodium phosphate was 
purchased from the National Chemical Reagent Co. (Chongqing, China). All the other reagents were obtained from J\&K Chemical Reagent Co., Ltd. Solutions were prepared with double deionized water $(18.2 \mathrm{M} \Omega \mathrm{cm})$.

\subsection{Analysis}

Samples of $3 \mathrm{~mL}$ were withdrawn at predetermined time intervals form the reactor. Then $3 \mathrm{~mL}$ of MTBE was added as an extraction solvent. After that, the mix was vigorously shaken for $2 \mathrm{~min}$, and left idle for $4 \mathrm{~min}$. The MTBE layer was transferred into a $2 \mathrm{~mL}$ amber vial. One microliter of the solvent was injected into a GC-ECD equipped with HP-5 capillary column (Agilent 7890A, USA) according to EPA.551.1.9 The temperature of injector was programed at $150{ }^{\circ} \mathrm{C}$. The initial column temperature was at $40{ }^{\circ} \mathrm{C}$ and rose to $240{ }^{\circ} \mathrm{C}$ at $40{ }^{\circ} \mathrm{C} \mathrm{min} \mathrm{m}^{-1}$ afterwards, then remained at $240{ }^{\circ} \mathrm{C}$ for $1 \mathrm{~min}$. The temperature of detector was at $250{ }^{\circ} \mathrm{C}$. The retention time of DCAN was $3.02 \mathrm{~min}$ and extraction efficiency was about $55.7 \%$. The $\mathrm{pH}$ of solution was determined by a pH meter (Hach HQ11D, USA).

\subsection{Experimental procedures}

The phosphate buffer was used to maintain the $\mathrm{pH}$ of the reaction at around 7.0. The UV lamp (GPH212T5L/4, $254 \mathrm{~nm}$, $10 \mathrm{~W}$, Heraeus, Germany) with a quartz sleeve was inserted vertically into the reactor to ensure the reaction system was radiated uniformly. The initial concentration of DCAN was set at $2 \mu \mathrm{M}$. A water bath was applied to keep the temperature of reaction at about $25{ }^{\circ} \mathrm{C}$. there was a magnetic stirrer (400 rpm) at the bottom of the reactor to mix the reaction. Diffident length of tin foil paper was used to cover the UV lamp to adjust the UV power inputted to the reaction.

\subsection{Deign of experiment with response surface methodology}

The RSM based on Box-Behnken Design (BBD) was employed to investigate the interactions of three factors though seventeen sets of experiments. The ranges and levels of three variables was shown in Table 1. A second-order polynomial equation was applied in date analysis to response the three variables according to eqn (1). ${ }^{\mathbf{1 0}}$

$$
Y=\beta_{0}+\sum_{i=1}^{n} \beta_{i} x_{i}+\sum_{i=1}^{n} \beta_{i i} x_{i}^{2}+\sum_{i=1}^{n-1} \sum_{j=i+1}^{n} \beta_{i i} x_{i} x_{j}
$$

$Y$ is the predicated response (degradation efficiency of DCAN). $\beta_{i}, \beta_{i i}$ and $\beta_{i j}$ are regression coefficients and $\beta_{0}$ is the interception coefficient. The data analysis including variance

Table 1 Ranges and levels of the variables for experimental design

\begin{tabular}{llllll}
\hline & & & \multicolumn{3}{l}{ Ranges and levels } \\
\cline { 4 - 5 } Variables & Code & Unit & -1 & 0 & 1 \\
\hline \multirow{2}{*}{ Dosage of PMS } & A & mM & 0.2 & 0.35 & 0.5 \\
UV power & B & W & 2 & 6 & 10 \\
Retention time & C & min & 20 & 50 & 80
\end{tabular}

(ANOVA) and proper regression equation was obtained though Design Expert 8.05b. Moreover, response surfaces and optimal condition of the process were obtained afterwards.

\section{Results and discussion}

\subsection{Effect of factors}

The effect of dosage of PMS, UV power and $\mathrm{pH}$ were shown in Fig. 1-3. As present in Fig. 1, with the dosage of PMS increased from $0.2 \mathrm{mM}$ to $0.35 \mathrm{mM}$, the degradation efficiency of DCAN increased significantly. It was due to that more free radicals including $\mathrm{SO}_{4}{ }^{-\cdot}$ and $\cdot \mathrm{OH}$ were produced by the increase of PMS dosage (eqn (2)), leading to the acceleration of the degradation. However, the degradation efficiency remained almost the same value which the dosage of PMS increased from $0.35 \mathrm{mM}$ to $0.5 \mathrm{mM}$. It could be explained that self-quenching (eqn (3) and (4)) and self-combination (eqn (5) and (6)) of free radicals was occurred, because of excess PMS in the system. ${ }^{11}$ Therefore, further increasing the dosage of PMS has negligible significance for improving the degradation efficiency.

$$
\begin{gathered}
\mathrm{HSO}_{5}{ }^{-} \stackrel{h v}{\longrightarrow} \mathrm{SO}_{4}^{-} \cdot+\cdot \mathrm{OH} \\
\mathrm{HSO}_{5}{ }^{-}+\mathrm{SO}_{4}^{-\cdot} \rightarrow \mathrm{SO}_{5}^{-\cdot}+\mathrm{SO}_{4}^{2-}+\mathrm{H}^{+} \\
\mathrm{HSO}_{5}^{-}+\mathrm{HO}^{\cdot} \rightarrow \mathrm{SO}_{5}^{-\cdot}+\mathrm{H}_{2} \mathrm{O} \\
\mathrm{SO}_{4}^{-\cdot}+\mathrm{SO}_{4}^{-\cdot} \rightarrow \mathrm{S}_{2} \mathrm{O}_{8}^{2-} \\
\mathrm{HO}^{-}+\mathrm{HO}^{-} \rightarrow \mathrm{H}_{2} \mathrm{O}_{2}
\end{gathered}
$$

Fig. 2 showed the effect of UV power on DCAN degradation efficiency. With UV power increased from $2 \mathrm{~W}$ to $6 \mathrm{~W}$, the degradation efficiency of DCAN increased about $24 \%$ after $30 \mathrm{~min}$. However, the degradation rate even had a slight decline with UV power increased from $6 \mathrm{~W}$ to $10 \mathrm{~W}$ in the first period of reaction. It might be because excessive UV power accelerated the photolysis of PMS, which made the radicals excessive, resulting the self-quenching of radicals.

Due to the hydrolysis, DCAN was unstable while in alkaline conditions. ${ }^{12}$ Thus, the effect of $\mathrm{pH}$ on the degradation was

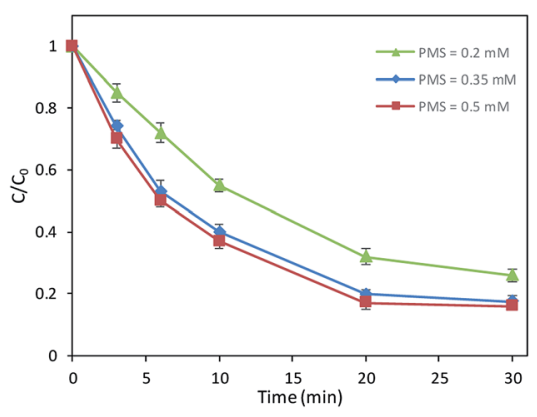

Fig. 1 Effects of PMS dosage on DCAN degradation. Conditions: initial concentration of DCAN $=2 \mu \mathrm{M}$, temperature $=25^{\circ} \mathrm{C}, \mathrm{pH}=7.0$, UV power $=10 \mathrm{~W}$. 


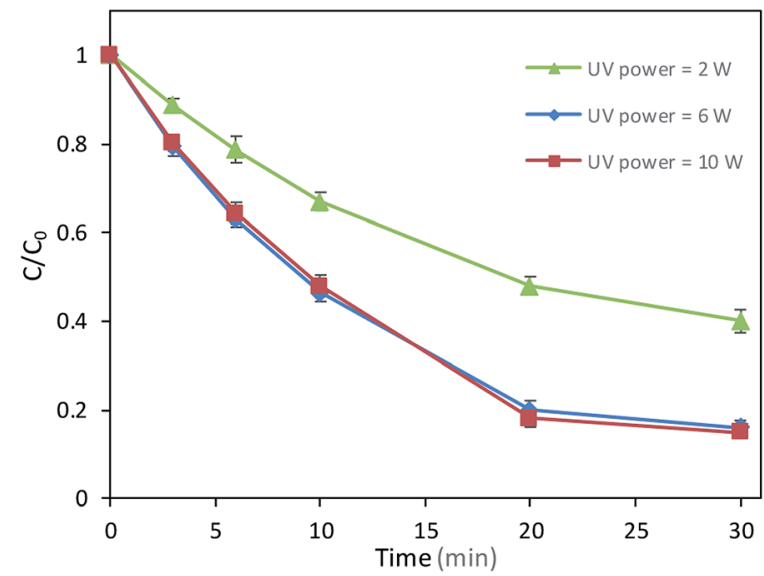

Fig. 2 Effects of UV power on DCAN degradation. Conditions: initial concentration of DCAN $=2 \mu \mathrm{M}$, temperature $=25^{\circ} \mathrm{C}, \mathrm{pH}=7.0, \mathrm{PMS}=$ $0.5 \mathrm{mM}$.

examined with $\mathrm{pH}$ ranging from 3.0 to 7.0. As shown in Fig. 3, the degradation efficiency was $77 \%$ at $\mathrm{pH} 7.0$ after $30 \mathrm{~min}$ reaction, which increased slightly to $83 \%$ and $90 \%$ with the $\mathrm{pH}$ of solution at 5.0 and 3.0, respectively. It indicated the degradation rate was slightly faster in acidic condition than that in neutral condition. It was consistent with previous research on the degradation of sucralose. ${ }^{13}$ The reason might be that the oxidation capacity of $\cdot \mathrm{OH}$ deceased with increasing $\mathrm{pH}$ value. . $\mathrm{OH}$ has a standard redox potential of $2.7 \mathrm{~V}$ in acidic $\mathrm{pH}$ condition and $1.8 \mathrm{~V}$ in neutral $\mathrm{pH}$ condition. ${ }^{\mathbf{1 4}}$

The target of the oxidization process was DCAN. It was high cytotoxic and genotoxic and frequently found in drinking water. Consequently, UV/PMS process studied in this paper was aimed at drinking water which kept neutral in most cases. Moreover, it is hard to adjust $\mathrm{pH}$ in the practical drinking water treatment. Based on this, the effect of $\mathrm{pH}$ was not discussed in the RSM design and the $\mathrm{pH}$ of solution was kept around 7.0 though phosphate buffer in the experiments afterwards.

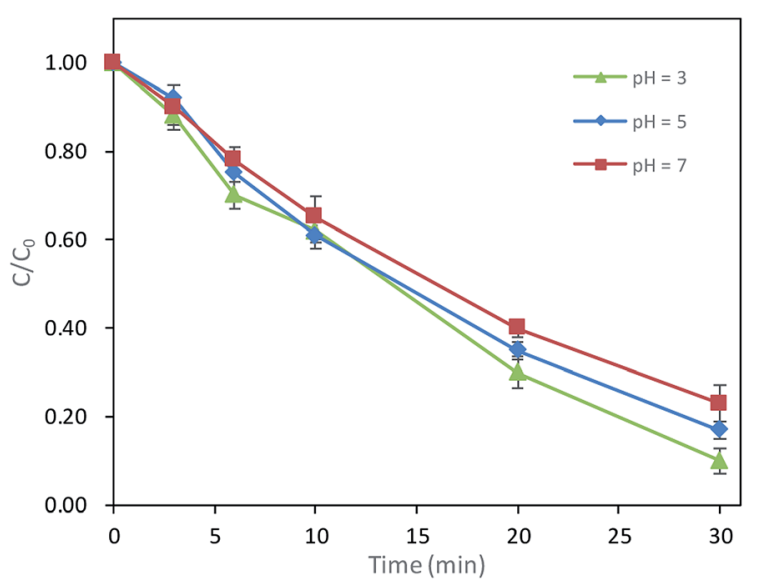

Fig. 3 Effects of $\mathrm{pH}$ on DCAN degradation. Conditions: initial concentration of DCAN $=2 \mu \mathrm{M}$, temperature $=25^{\circ} \mathrm{C}$, PMS $=0.4 \mathrm{mM}$, UV power $=6 \mathrm{~W}$.

\subsection{Model fitting and statistical analysis}

According to Box-Behnken design, the batch runs was executed and the values of experiment and predicated values were present in Table 2 . The regression equation was obtain as following:

$$
\begin{gathered}
Y=-87.44+207.78 A+19.52 B+1.83 C-11.08 A B \\
-1.489 A C-0.08 B C-28.33 A^{2}-0.67 B^{2}-3.40 \times 10^{-3} C^{2}
\end{gathered}
$$

where $A, B$ and $C$ represent dosage of PMS, UV power and retention time, respectively. $Y$ is the degradation efficiency of DCAN.

The experimental values of 17 experiments (shown in Table 2) were inputted to Design Expert 8.05b software. With the model fitting and error calculation, Table 3 was outputted by the software. The results of ANOVA in DCAN degradation were shown in Table 3 . The $F$-value and value of prob. $>F$ were pivotal index for model evaluation. ${ }^{15} \mathrm{~A}$ high $F$-value $(F$-value $=95)$ and a low value of prob. $>F$ (prob. $>F<0.05$ ) of the model indicated the model was significant. The $F$-value of lack of fit was 2.9, which suggested the lack of fit was not significant relative to the pure error. Adeq. precision meant the signal to noise ratio. A value of the ratio greater than 4 was desirable. Adeq. precision of this model was reached to 34.31 , which was an adequate signal for the model.

Moreover, the $R^{2}$ of 0.9919 and $\operatorname{Adj}-R^{2}$ of 0.9814 indicated the model fitted well with the experiment values. Values of prob. $>F$ less than 0.05 indicate model terms are significant and the influence of model term was positively correlated with the value of $F$-value. In this model, $A, B, C, A B, A C, B C, B^{2}$, and $C^{2}$ were significant model terms, which indicated they had a great influence on the degradation efficiency of DCAN in UV/PMS process. Additionally, the order of the influence among variables followed: $C>B>B^{2}>B C>A>A C>A B>C^{2}$. Values greater

\begin{tabular}{|c|c|c|c|c|c|}
\hline \multirow[b]{2}{*}{ Run } & \multicolumn{3}{|l|}{ Variables } & \multicolumn{2}{|c|}{$\begin{array}{l}\text { Degradation } \\
\text { efficiency of } \\
\operatorname{DCAN}(Y, \%)\end{array}$} \\
\hline & PMS (mM) & $\mathrm{UV}(\mathrm{W})$ & Time (min) & Actual & Predicted \\
\hline 1 & 0.35 & 6 & 50 & 82.0 & 83.6 \\
\hline 2 & 0.35 & 2 & 80 & 78.4 & 80.2 \\
\hline 3 & 0.35 & 6 & 50 & 81.5 & 83.6 \\
\hline 4 & 0.5 & 6 & 80 & 95.0 & 94.4 \\
\hline 5 & 0.35 & 6 & 50 & 84.5 & 83.6 \\
\hline 6 & 0.35 & 10 & 20 & 81.2 & 79.5 \\
\hline 7 & 0.35 & 6 & 50 & 84.4 & 83.6 \\
\hline 8 & 0.35 & 2 & 20 & 30.2 & 31.9 \\
\hline 9 & 0.35 & 6 & 50 & 85.6 & 83.6 \\
\hline 10 & 0.2 & 6 & 20 & 51.4 & 52.0 \\
\hline 11 & 0.2 & 10 & 50 & 84.5 & 85.6 \\
\hline 12 & 0.2 & 6 & 80 & 93.1 & 93.7 \\
\hline 13 & 0.5 & 10 & 50 & 84.1 & 86.4 \\
\hline 14 & 0.5 & 2 & 50 & 73.3 & 72.2 \\
\hline 15 & 0.35 & 10 & 80 & 89.5 & 87.8 \\
\hline 16 & 0.5 & 6 & 20 & 80.1 & 79.5 \\
\hline 17 & 0.2 & 2 & 50 & 47.1 & 44.8 \\
\hline
\end{tabular}

Table 2 Experimental design matrix and the value of responses 
Table 3 Analysis of variance for the quadratic model of DCAN degradation by UV/PMS process

\begin{tabular}{lccrrr}
\hline Source & Sum of squares & df & Mean square & $F$-value & Prob. $>F$ \\
\hline Model & 4818.83 & 9 & 535.43 & 95.00 & $<0.0001$ \\
$A$-dosage of PMS & 190.37 & 1 & 190.37 & 33.78 & 0.0007 \\
$B$-UV power & 876.33 & 1 & 876.33 & 155.49 & $<0.0001$ \\
$C$-retention time & 927.55 & 1 & 927.55 & 164.57 & $<0.0001$ \\
$A B$ & 176.89 & 1 & 176.89 & 31.39 & 0.0008 \\
$A C$ & 179.56 & 1 & 179.56 & 31.86 & 0.0008 \\
$B C$ & 398.00 & 1 & 398.00 & 0.30 & 0.0001 \\
$A^{2}$ & 1.71 & 1 & 1.71 & 85.73 & $<0.0001$ \\
$B^{2}$ & 483.19 & 1 & 483.19 & 7.01 & 0.0331 \\
$C^{2}$ & 39.49 & 1 & 39.49 & & \\
Residual & 39.45 & 7 & 5.64 & 2.90 & 0.1650 \\
Lack of fit & 27.03 & 3 & 9.01 & & Not significant \\
Pure error & 12.42 & 4 & 3.11 &
\end{tabular}

than 0.10 indicate the model terms are not significant, indicated $\mathrm{A}^{2}$ had negligible influence in DCAN degradation.

The predicated value matched well with experimental value, shown in Fig. 4. It indicated the model fitted well with the actual process. The internally studentized residuals were investigated, as shown in Fig. 5. The plots of residuals were nearly at a straight line, which further proved predicated values and experimental values were almost consistent. ${ }^{\mathbf{1 6}}$ The plots of residuals versus model predictions and was shown in Fig. 6. As shown in Fig. 6, the plots were distributed randomly, indicating the variance of environmental was a constant value. It suggested that the model was adequate. ${ }^{\mathbf{1 7}}$ Based on result above, it could stated that the model simulated the actual condition well and the degradation process of DCAN by UV/PMS could be analyzed and explained by this model.

\subsection{Analysis of response surface}

Fig. 7 showed the effects of UV power and PMS dosage on the degradation efficiency while the reaction time was kept at 50 min as a constant. As shown in Fig. 7, at the low dosage of

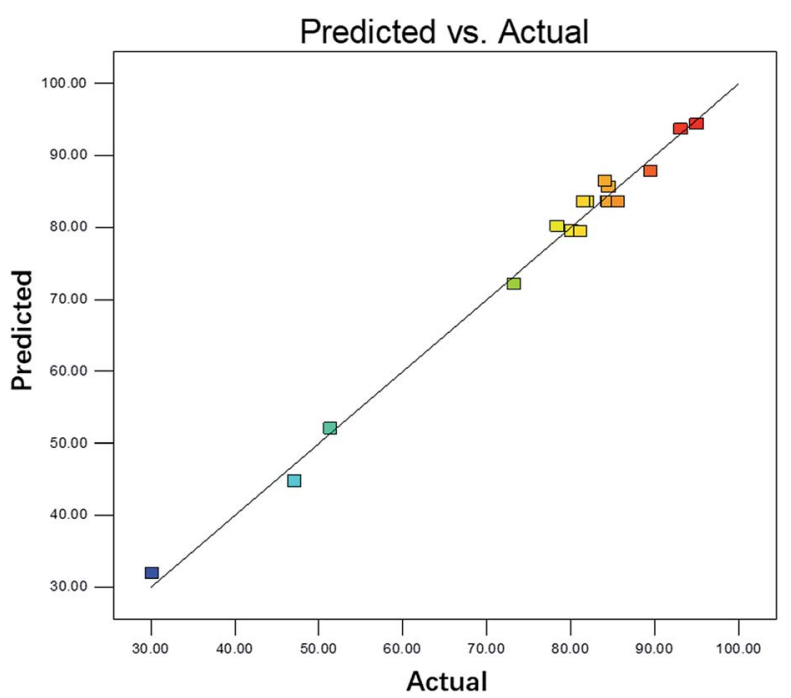

Fig. 4 Plot of predicted value versus experimental value.
PMS, the degradation efficiency increased visibly with the increase of UV power. However, while the dosage of PMS was at a high level (about greater than $0.44 \mathrm{mM}$ ), the degradation efficiency increased first and decreased afterwards with the increase of UV power. This phenomenon might be caused by self-quenching and self-combination of free radicals. With the UV power increasing, the decomposition efficiency of PMS increased, which made the free radicals generated by UV/PMS system increased. At the low dosage of PMS, the sulfate radicals and hydroxyl radicals which could be generated by the system were limited. Even if the PMS added in system was completely decomposed, the amount of radicals generated was still relatively small due to the low dosage of PMS. However, with the PMS dosage being at a high level, the decomposition efficiency of PMS was too fast when the UV power continued to increase (about more than $8 \mathrm{~W}$ ). Self-quenching (eqn (3) and (4)) and self-combination (eqn (5) and (6)) of free radicals was occurred, which reduced the amount of free radicals, resulting the decrease of the degradation efficiency.

The effects of reaction time and PMS dosage on the degradation efficiency were shown in Fig. 8, with UV power kept at

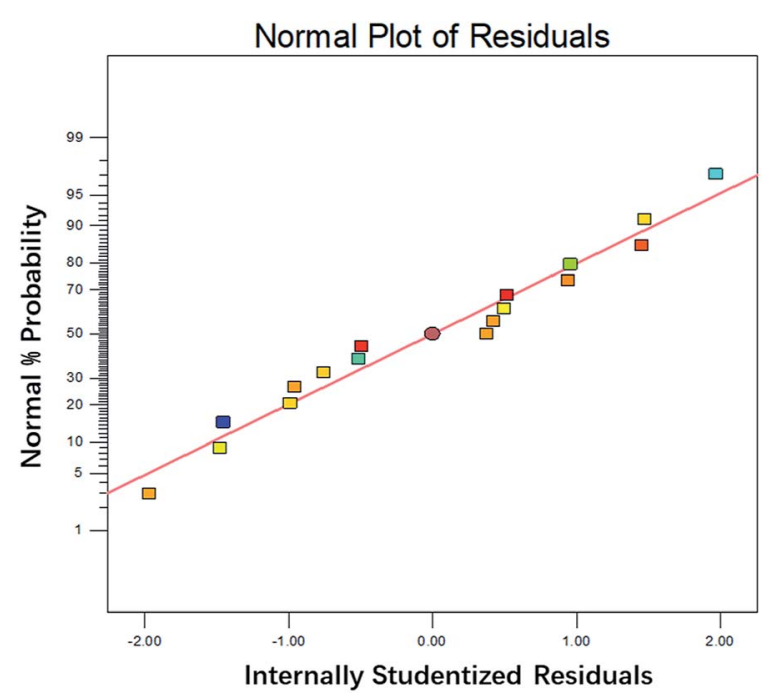

Fig. 5 The normal plot of residual for 17 experimental values designed by RSM. 


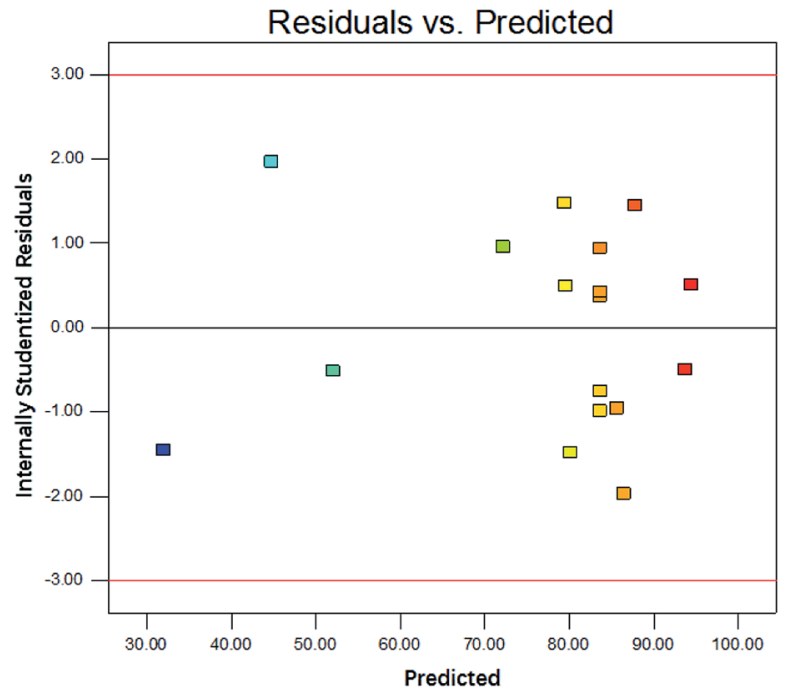

Fig. 6 Plot of the residual versus model predictions for the DCAN degradation.

6 W. As shown, the degradation efficiency increased with increase of reaction time and PMS dosage. While the reaction time was short, the degradation efficiency increased rapidly with the increase of PMS dosage; when the reaction time was longer, the degradation efficiency increased little with the increase of PMS dosage. It could be explained that PMS added in the reaction was not decomposed completely, and the amount of free radicals in the reaction system was relatively small when the reaction time was short. In this condition, the dosage of PMS determined the amount of free radicals and played a crucial role in the degradation efficiency. However, when the reaction time was long enough, PMS added was fully decomposed. The number of free radicals was much larger than the number of DCAN molecules. In this condition, the number of free radicals was not the main influencing factor. As a result, the degradation efficiency was not sensitive to the dosage of PMS.
Fig. 9 presented the effects of UV power and reaction time on the degradation efficiency with PMS dosage kept at $0.4 \mathrm{mM}$. As shown in Fig. 9, when the reaction time was short, the degradation efficiency increased with the increase of UV power. When the reaction time was longer, the degradation efficiency increased initially and decreased afterwards with the increase of UV power, which was similar to that in Fig. 7. For a long reaction time and high UV power, PMS decomposed quickly, generating a large amount of free radicals. The quenching reaction (eqn (3) and (4)) and self-combination (eqn (5) and (6)) of occurred due to excess free radicals, which leaded to an inhibition on the degradation efficiency.

Based on Table 3 and Fig. 7-9, the influence of factors was followed: retention time > UV power $>$ dosage of PMS. The conclusion could be a guide for an actual project. In the design of water treatment equipment of UV/PMS process, compared with dosage of PMS, the hydraulic retention time and UV power should be given more priority. Moreover, in actual engineering, experiments should be conducted on UV lamps to obtain the proper UV power, preventing setting excessive UV power and self-quenching and self-combination of free radicals.

\subsection{Optimization of the operating parameters and verification of the model}

According to the optimization function of Design Expert 8.05b, the optimum condition of this model was suggested though the derivation of the quadratic polynomial model equation and scope of the three independent variables (PMS dosage, UV power and retention time). The degradation efficiency would reach the maximum value of $96.2 \%$ with the optimum condition: dosage of PMS $=0.2 \mathrm{mM}$, UV power $=7.95 \mathrm{~W}$, retention time $=80 \mathrm{~min}$. To verify the model prediction, three sets of parallel experiments were carried out under the optimal conditions of model optimization. The average of degradation efficiency was $95.7 \%$, which was almost consistent with the
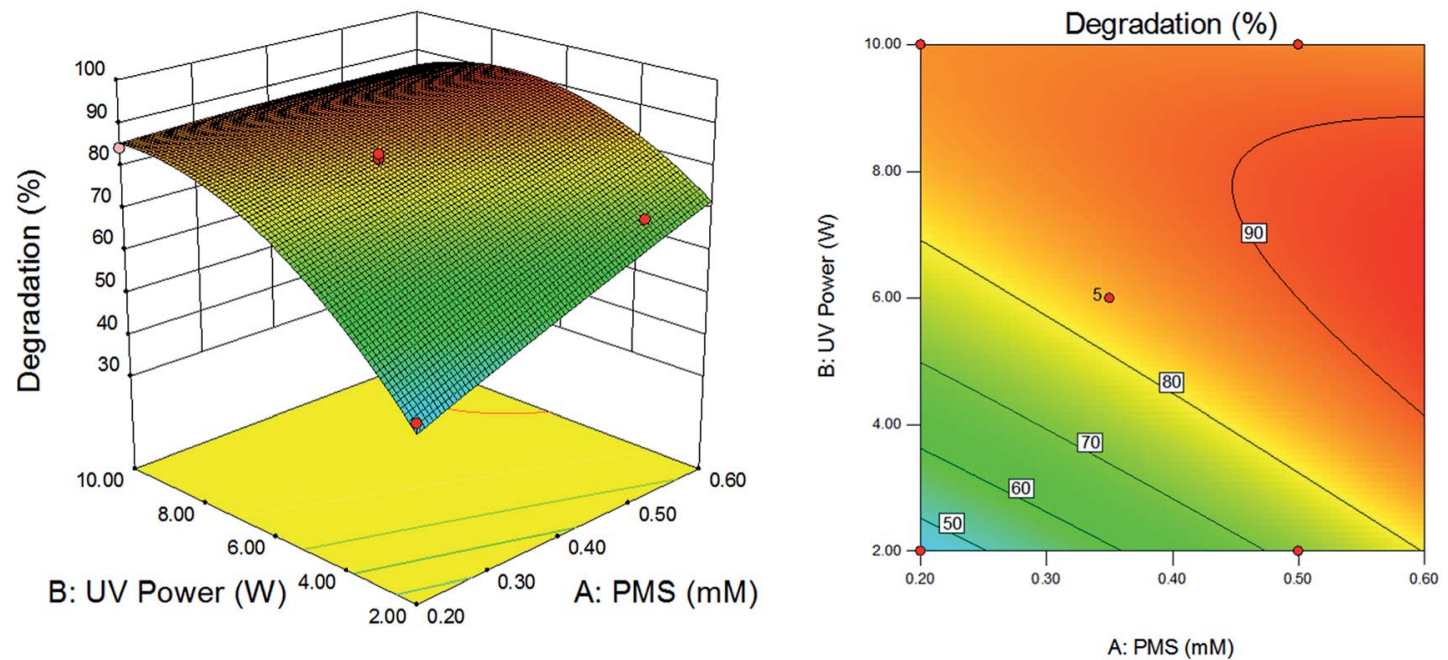

Fig. 7 Effects of dosage of PMS and power of UV on degradation efficiency of DCAN. Retention time: 50 min. 

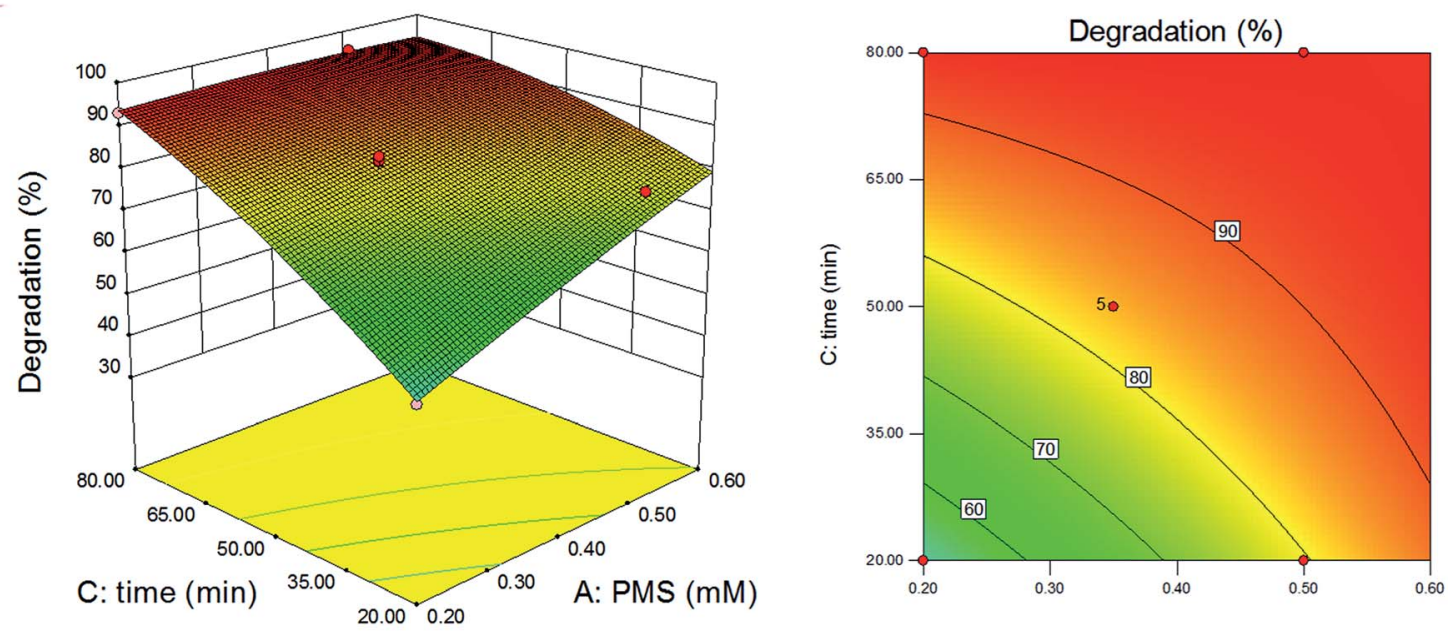

A: PMS (mM)

Fig. 8 Effects of dosage of PMS and retention time on degradation efficiency of DCAN. Power of UV: $6 \mathrm{~W}$.
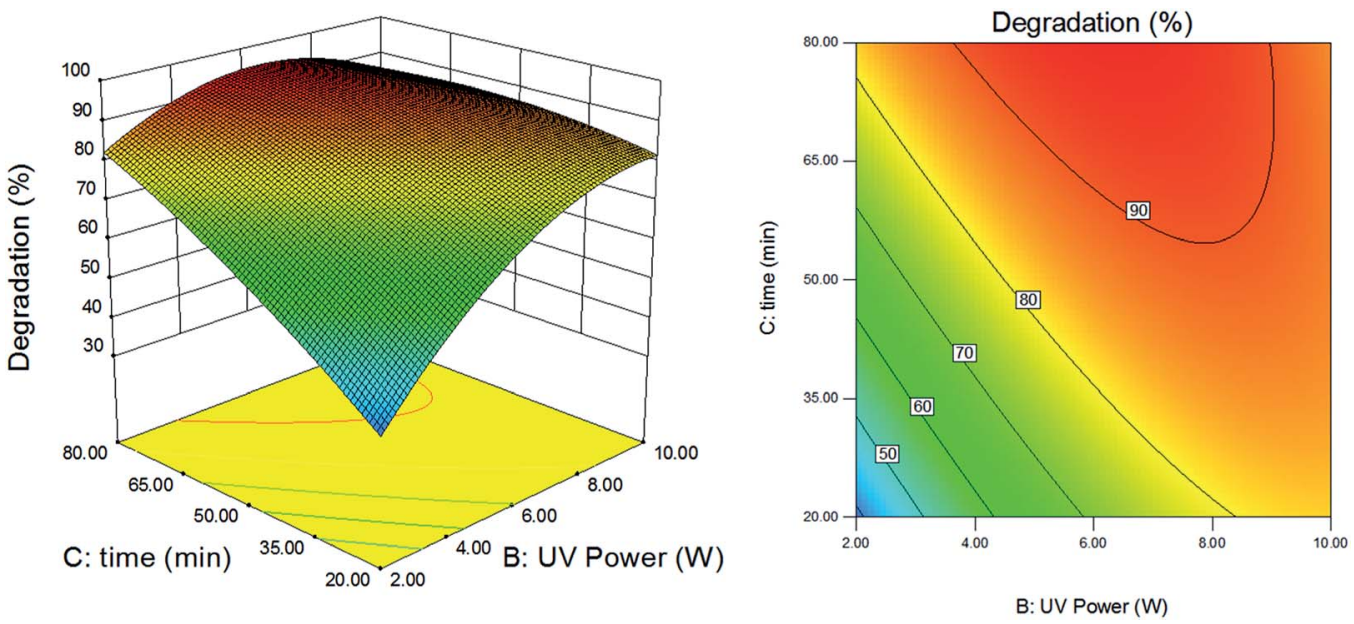

Fig. 9 Effects of power of UV and retention time on degradation efficiency of DCAN. Dosage of PMS: 0.4 mM.

predicted value of the model. The reliability of the model was successfully verified. It could be concluded that RSM could be used to optimize the process of DCAN degradation by UV/PMS.

\section{Conclusion}

In this study, RSM was utilized to model and optimize the dichloroacetonitrile (DCAN) degradation process by UV/PMS. The model fitted well with the experiment data according to high value of $R^{2}(0.9919)$ and Adj- $R^{2}(0.9814)$. The effects of PMS dosage, UV, residence time and interaction effects were also studied. Based on ANOVA results, the influence of factors on degradation efficiency was followed: retention time $>$ UV power $>$ dosage of PMS. The quadratic function relationship between the degradation efficiency and the three influencing factors was given. Under optimal conditions $(\mathrm{PMS}=0.2 \mathrm{mM}$, UV power $=$ $7.95 \mathrm{~W}$, retention time $=80 \mathrm{~min}$ ), the degradation efficiency reached the maximum value of $96.2 \%$, which was consistent with the experimental value of $95.7 \%$. In a conclusion, this work showed that the model based on RSM could be utilized to study and optimize the process of DCAN degradation by UV/PMS.

\section{Conflicts of interest}

There are no conflicts to declare.

\section{Acknowledgements}

We are thankful for the financial support from the National key research and development plan of China (Grant no. 2017YFC0806305), the National Natural Science Foundation of China (Grant no. 51508564).

\section{References}

1 J. J. Rook, Water Treat. Exam., 1974, 23, 234-243. 
2 M. G. Muellner, E. D. Wagner, K. McCalla, S. D. Richardson, Y. Woo and M. J. Plewa, Environ. Sci. Technol., 2007, 41, 645651.

3 S. W. Krasner, H. S. Weinberg, S. D. Richardson, S. J. Pastor, R. Chinn, M. J. Sclimenti, G. D. Onstad and A. D. Thruston, Environ. Sci. Technol., 2006, 40, 7175-7185.

4 D. Baytak, A. Sofuoglu, F. Inal and S. C. Sofuoglu, Sci. Total Environ., 2008, 407, 286-296.

5 W. H. Organization, Guidelines for Drinking-water Quality, World Health Organization, 2011, ISBN 978-92-4-155001-7.

6 S. Ding, W. Chu, T. Bond, Q. Wang, N. Gao, B. Xu and E. Du, J. Hazard. Mater., 2018, 341, 112-119.

7 S. Hou, L. Ling, C. Shang, Y. Guan and J. Fang, Chem. Eng. J., 2017, DOI: 10.1016/j.cej.2017.03.042.

8 P. Kiattisaksiri, E. Khan, P. Punyapalakul and T. Ratpukdi, Water Res., 2016, 98, 160.

9 U. S. EPA, Determination of chlorination disinfection byproducts, chlorinated solvents, and halogenated pesticides/ herbicides in drinking water by liquid-liquid extraction and gas chromatography with electron-capture detection (Revision 1.0), 1995.
10 L. C. Almeida and S. Garcia-Segura, Appl. Catal., B, 2011, 103, 21-30.

11 J. A. Khan, X. He, H. M. Khan, N. S. Shah and D. D. Dionysiou, Chem. Eng. J., 2013, 218, 376-383.

12 N. Gao, J. Huazhong Univ. Sci. Technol., Med. Sci., 2011, 39, 111-114.

13 Y. Xu, Z. Lin, Y. Wang and H. Zhang, Chem. Eng. J., 2017, 317, 561-569.

14 G. V. Buxton, C. L. Greenstock, W. P. Helman and A. B. Ross, J. Phys. Chem. Ref. Data, 1988, 17, 513-886.

15 S. Bose and C. Das, Ind. Eng. Chem. Res., 2014, 53, 1231912329.

16 R. H. Myers, D. C. Montgomery and C. M. Anderson-Cook, Response Surface Methodology: Process and Product Optimization Using Designed Experiments, Wiley, Hoboken 2009.

17 L. Y. Cherif, I. Yahiaoui, F. Aissanibenissad, K. Madi, N. Benmehdi, F. Fourcade and A. Amrane, Ind. Eng. Chem. Res., 2014, 53, 3813-3819. 